\title{
The impact of COVID-19 on ophthalmology practice in Egypt
}

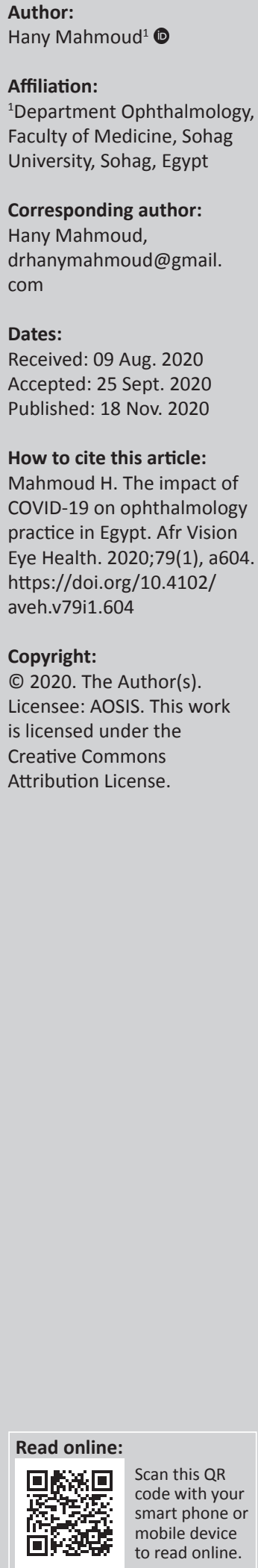

Background: The emergence of coronavirus disease 2019 (COVID-19) affected medical practice all over the world including ophthalmology, especially elective procedures.

Objectives: Evaluation of the effect of COVID-19 on ophthalmology practice in Egypt.

Method: A cross-sectional study was done using an internet-based questionnaire including Egyptian ophthalmologists.

Results: A total of 1257 participants completed the questionnaires. A majority of the responders $(79.9 \%)$ were working in private ophthalmic organisations. Out of $(79.9 \%)$ responders, $(57.5 \%)$ were seeing patients at the time of the survey. The majority of patients seen $(32 \%)$ and operations done $(21.1 \%)$ were both emergency and electives. Most of the responders $(82 \%)$ did not try to use telemedicine. The majority of the participants $(81.5 \%)$ thought that the lockdown had a detrimental effect on the progression and follow-up of chronic diseases (e.g. glaucoma).

Conclusion: COVID-19 affects elective ophthalmology practice; we need to activate the role of telemedicine according to ethics.

Keywords: COVID-19; lockdown; ophthalmology; ophthalmology practice; ophthalmology in Egypt.

\section{Introduction}

Since the emergence of severe acute respiratory syndrome (SARS-CoV-2) and the mandatory lockdown all over the world with limitation of many activities to decrease transmission of the coronavirus disease 2019 (COVID-19) infection ${ }^{1,2}$ medical practice has been affected; all facilities are directed to fight COVID-19 with cancellation or reduction of other medical services, especially elective ones. ${ }^{3}$

Ophthalmology is a medical discipline that is elective in most of its practice away from ocular trauma or other few emergency conditions, so many ophthalmology services are affected in the world. This was to concentrate efforts to fight COVID-19 at first and decrease infection amongst ophthalmologists as the virus could be transmitted by conjunctival secretions. Many studies proved that the virus ribonucleic acid (RNA) could be detected in tears and conjunctival secretions. ${ }^{4,5}$

In many countries, ophthalmology practice could be private or governmental, and the lockdown with fear of infection affected both. Many ophthalmologists have stopped their service either by law or self-decision. ${ }^{6}$

In some countries with well-established electronic infrastructure and clear laws for telemedicine, some ophthalmology services remain especially for the follow-up of chronic diseases. When this service was absent, the patients were severely affected by deterioration in their conditions, especially in patients suffering from glaucoma or diabetic retinopathy. ${ }^{8}$

However, ophthalmologists are still practicing ophthalmology, but they are restricted to emergency situations with full infection control measures. ${ }^{9}$ Surgical practice is restricted to those operations that threaten vision ${ }^{10}$ such as trauma, acute congestive glaucoma or fresh retinal detachment with strict infection control precautions.

Telemedicine as a new technology needs special infrastructure with law regulations, so its usage was restricted ${ }^{11}$; we need to encourage it to avoid over crowdedness in hospitals and private practice. Doctors and patient awareness are both needed for telemedicine applications. ${ }^{12}$

During lockdown and lack of medical services, patients with chronic diseases who needed continuous follow-up were severely affected and deteriorated, such as glaucoma patients who needed continuous monitoring of intraocular pressure. ${ }^{13}$ 
Patients with diabetic retinopathy were affected as emergent situations failed to find appropriate service because of stopping practice either by law or by self-decision and some ophthalmologists shared in guarantee services in guarantee hospitals.

\section{Aim of the study}

To evaluate the effects of COVID-19 lockdown on ophthalmology practice in Egypt.

\section{Materials and methods}

A cross-sectional study was conducted amongst Egyptian ophthalmologists in June 2020. The survey included ophthalmologists from both governmental and private ophthalmic organisations in Egypt. We designed an internetbased questionnaire survey (Figure 1) addressing the issue in six separate questions.

\section{Ethical consideration}

The author confirms that ethical clearance was not required for the study. Ethical considerations were followed according to the Helsinki Declaration. Anotification was in the questionnaire about accepting to participate in the questionnaire or refusing it, and this was considered as a providing consent.

\section{Results}

The survey results are shown in Figure 1. A total of 1257 participants completed the questionnaires. A majority of the responders $(79.9 \%)$ were working in private ophthalmic organisations. A total of $57.5 \%$ of the responders were seeing patients at the time of the survey. The majority of patients seen $(32 \%)$ and operations done $(21.1 \%)$ were both emergency and electives.

Most of the responders (82\%) did not try to use telemedicine. The majority of the participants $(81.5 \%)$ thought that the lockdown had a detrimental effect on the progression and follow-up of chronic diseases (e.g. glaucoma).

\section{Discussion}

However, ophthalmology practice is affected but some still examine patients either in governmental or private practice; we think that examining patients in governmental practice, especially emergency cases was enforced by regulations and laws. This occurred in many countries.

Some ophthalmologists limited their practice to emergencies only, but others still examined elective patients with full infection control measures, including personal protective equipment (PPE) to avoid COVID-19 infection. ${ }^{9}$ A large percentage of ophthalmologists limited their surgical practice to emergency situations that threaten vision only, such as trauma, fresh retinal detachment or acute congestive glaucoma. Elective eye problems or those who need close or frequent follow-up were severely affected. ${ }^{10}$

Telemedicine limitations such as lack of infrastructure, experience, patient awareness or doctor experience could explain the lack of its use in practice either before or during the lockdown.

Patients who require frequent follow-up, such with glaucoma or diabetic retinopathy, were severely affected with lack of self-examining tools and technologies.

According to the questionnaire, we should develop and enforce new technologies such as telemedicine and increase the awareness of this technology amongst health providers and the patients. ${ }^{11,12,13}$

\section{Conclusion}

Ophthalmology practice was affected in Egypt during the COVID-19 pandemic and the need for telemedicine emerged as a possible solution. Patients with elective situations were severely affected, but those with emergent problems were still served. We are in need to apply new technologies such as telemedicine with awareness to enforce ophthalmology practice during pandemic or urgent situations.

\begin{tabular}{|c|c|c|c|c|}
\hline \multirow[t]{2}{*}{ 1. Please specify the type of your ophthalmic organisation. } & Governmental & Private & & \\
\hline & $253(20.1)$ & 1004 (79.9) & - & - \\
\hline \multirow[t]{2}{*}{ 2. Are you currently examining patients? } & Yes & No & - & - \\
\hline & $723(57.5)$ & $534(42.5)$ & - & - \\
\hline \multirow[t]{2}{*}{ 3. What kind of patients do you currently examine? } & Emergency only & Elective only & Both of them & None of them \\
\hline & $270(21.5)$ & $50(4)$ & $403(32)$ & $534(42.5)$ \\
\hline \multirow[t]{2}{*}{ 4. What kind of operations do you currently do? } & Emergency only & Elective only & Both of them & None of them \\
\hline & 250 (19.9) & $69(5.5)$ & $266(21.1)$ & $672(53.5)$ \\
\hline \multirow[t]{2}{*}{ 5. Have you tried using telemedicine? } & Yes & No & - & - \\
\hline & $226(18)$ & $1031(82)$ & - & - \\
\hline \multirow[t]{2}{*}{ 6. What was the effect of lockdown on the progression of chronic diseases (e.g. glaucoma)? } & Deteriorated & Improved & Did not change & - \\
\hline & $1024(81.5)$ & $9(0.7)$ & $224(17.8)$ & - \\
\hline
\end{tabular}

Values are $n(\%)$.

FIGURE 1: Showing the internet-based questionnaire and its results. 


\section{Limitations}

Although there were a large number of participants for the questionnaire, we still need more to explore more data. The specified answers in the questionnaire limited the detailed information obtained from the participants.

\section{Acknowledgements Competing interests}

The author has declared that no competing interests exists.

\section{Author's contributions}

I declare that I am the sole author of this research article.

\section{Funding information}

This research received no specific grant from any funding agency in the public, commercial or not for profit sectors.

\section{Data availability statement}

Data sharing is not applicable to this article.

\section{Disclaimer}

The views and opinions expressed in this article are those of the author and do not necessarily reflect the official policy or position of any affiliated agency of the author.

\section{References}

1. The novel coronavirus pneumonia emergency response epidemiology team. The epidemiological characteristics of an outbreak of 2019 novel coronavirus diseases (COVID-19) in China. Chin J Epidemiol. 2020;41(2):145-151. https://doi. org/10.3760/cma.j.issn.0254-6450.2020.02.003

2. Chen $P$, Mao L, Nassis GP, Harmer P, Ainsworth BE, Li F. Coronavirus disease (COVID-19): The need to maintain regular physical activity while taking precautions. J Sport Health Sci. 2020;9(2):103-104. https://doi.org/10.1016/j. jshs.2020.02.001

3. Khanna RC, Honavar SG. All eyes on coronavirus - What do we need to know as ophthalmologists. Indian J Ophthalmol. 2020;68(4):549-553. https://doi. org/10.4103/ijo.IJO_516_20

4. Lu CW, Liu XF, Jia ZF. 2019-nCoV transmission through the ocular surface must not be ignored. Lancet. 2020;395(10224):e39. https://doi.org/10.1016/S01406736(20)30313-5

5. Qing $H$, Li Z, Yang Z, et al. The possibility of COVID-19 transmission from eye to nose. Acta Ophthalmol. 2020;98(3):e388. https://doi.org/10.1111/aos.14412

6. Lai THT, Tang EWH, Chau SKY, Fung KSC, Li KKW. Stepping up infection control measures in ophthalmology during the novel coronavirus outbreak: An experience from Hong Kong. Graefes Arch Clin Exp Ophthalmol. 2020;258(5):1049-1055. https://doi.org/10.1007/s00417-020-04641-8

7. Hollander JE, Carr BG. Virtually perfect telemedicine for COVID-19. N Engl J Med. 2020;382:1679-1681. https://doi.org/10.1056/NEJMp2003539

8. Liebmann JM. Ophthalmology and glaucoma practice in the COVID-19 era. J Glaucoma. 2020;29(6):407-408. https://doi.org/10.1097/IJG.0000000000001519

9. Wan KH, Huang SS, Young A, Chiu Lam DS. Precautionary measures needed for ophthalmologists during pandemic of the coronavirus disease 2019 (COVID-19). Acta Ophthalmol. 2020;98(3):221-222. https://doi.org/10.1111/aos.14438

10. American Academy of Ophthalmology. List of urgent and emergent ophthalmic procedures [homepage on the Internet]. 2020 [cited 2020 Apr 05]. Available from: https://www.aao.org/headline/list-of-urgent-emergent-ophthalmic-procedures

11. Korot E, Wood E, Weiner A, Sim DA, Trese M. A renaissance of teleophthalmology through artificial intelligence. Eye. 2019;33(6):861-863. https://doi.org/10.1038/ s41433-018-0324-8

12. Healthcare IT News. Telemedicine during COVID-19: Benefits, limitations, burdens, adaptation [serial online]. 2020 [cited 2020 March 19]. Available from: https://www.healthcareitnews.com/news/telemedicine-during-covid-19benefits-limitations-burdens-adaptation

13. Gan K, Liu Y, Stagg B, et al. Telemedicine for glaucoma: Guidelines and recommendations. Telemed J E Health. 2020;26(4):551-555. https://doi. org/10.1089/tmj.2020.0009 